\title{
Comparison of Block Matching and Differential Methods for Motion Analysis of the Carotid Artery Wall From Ultrasound Images
}

\author{
Spyretta Golemati, Member, IEEE, John S. Stoitsis, Member, IEEE, Aimilia Gastounioti, Student Member, IEEE, \\ Alexandros C. Dimopoulos, Vassiliki Koropouli, and Konstantina S. Nikita, Senior Member, IEEE
}

\begin{abstract}
Motion of the carotid artery wall is important for the quantification of arterial elasticity and contractility and can be estimated with a number of techniques. In this paper, a framework for quantitative evaluation of motion analysis techniques from B-mode ultrasound images is introduced. Six synthetic sequences were produced using 1) a real image corrupted by Gaussian and speckle noise of 25 and $15 \mathrm{~dB}$, and 2) the ultrasound simulation package Field II. In both cases, a mathematical model was used, which simulated the motion of the arterial wall layers and the surrounding tissue, in the radial and longitudinal directions. The performance of four techniques, namely optical flow $\left(\mathrm{OF}_{\mathrm{HS}}\right)$, weighted least-squares optical flow $\left(\mathrm{OF}_{\mathrm{LK}(\mathrm{WLS})}\right)$, block matching (BM), and affine block motion model (ABMM), was investigated in the context of this framework. The average warping indices were lowest for $\mathrm{OF}_{L K}$ (W L S) (1.75 pixels), slightly higher for $\mathrm{ABMM}$ (2.01 pixels), and highest for $\mathrm{BM}$ (6.57 pixels) and $\mathrm{OF}_{\mathrm{HS}}$ (11.57 pixels). Due to its superior performance, $\mathrm{OF}_{\mathrm{LK} \text { (W LS) }}$ was used to quantify motion of selected regions of the arterial wall in real ultrasound image sequences of the carotid artery. Preliminary results indicate that $\mathrm{OF}_{L K}\left(\mathrm{WLS}_{\mathrm{L}}\right.$ ) is promising, because it efficiently quantified radial, longitudinal, and shear strains in healthy adults and diseased subjects.
\end{abstract}

Index Terms-Block matching (BM), carotid, motion analysis, optical flow (OF), ultrasound.

\section{INTRODUCTION}

$\mathbf{T}$ HE estimation of motion of the carotid artery wall is important for the quantification of arterial elasticity and contractility. The elasticity of the arterial wall decreases with age [1], and is altered in the presence of pathology (e.g., atherosclerosis) due to changes in its composition. Throughout the arterial tree, motion of the arterial wall is caused by blood pressure, blood flow, and tethering to surrounding tissue [2]. Blood pressure induces compressive stresses in the radial direction, and tensile stresses in the longitudinal and circumferential directions

Manuscript received October 1, 2011; revised February 16, 2012; accepted March 23, 2012. Date of publication April 4, 2012; date of current version September 20,2012. The work of A. Gastounioti was supported by a scholarship from the Hellenic State Scholarships Foundation.

S. Golemati is with the First Intensive Care Unit, Medical School, National and Kapodistrian University of Athens, Athens 10679, Greece (e-mail: sgolemati@biosim.ntua.gr).

J. S. Stoitsis, A. Gastounioti, A. C. Dimopoulos, V. Koropouli, and K. S. Nikita are with the Biomedical Simulations and Imaging Laboratory, School of Electrical and Computer Engineering, National Technical University of Athens, Athens 15780, Greece (e-mail: stoitsis@biosim.ntua.gr; gaimilia@biosim.ntua.gr; alexdem@cslab.ece.ntua.gr; vicky@1sr.ei.tum.de; knikita@ece.ntua.gr).

Digital Object Identifier 10.1109/TITB.2012.2193411 leading to tissue lengthening. Fluid shear generated by blood flow acts tangentially upon the vascular wall and endothelium. Tether, a term used to describe the constraint imposed on the arterial wall from the tissues surrounding it and from arterial side branches [2], reduces motion due to hemodynamic factors but can induce wall movement due to body motion.

Motion of the carotid artery wall can be quantitatively estimated from sequences of B-mode ultrasound images. Ultrasound imaging of the carotid artery is widely used in the diagnosis of atherosclerosis because it allows noninvasive assessment of the degree of stenosis as well as of tissue morphology. Tracking carotid artery wall motion in real time from a series of ultrasound images is challenging due to the fact that the images are noisy, images are acquired rapidly $(\approx 40 \mathrm{~ms})$, motion is nonrigid, and the image contrast/intensity may change over time due to flowing blood and/or contrast agent.

Block matching (BM) and optical flow (OF) have been previously used to estimate arterial tissue deformation during the cardiac cycle from ultrasound images using high frame rates. Golemati et al. [3] estimated motion at the wall-lumen interface and within the tissue using block sizes of $3.2 \times 2.5 \mathrm{~mm}$ $(50 \times 40$ pixels $)$ and $6.3 \times 2.5 \mathrm{~mm}(100 \times 50$ pixels $)$, respectively, and the normalized correlation coefficient as the matching criterion. Cinthio et al. used a small block size, namely $0.1 \times 0.1 \mathrm{~mm}(2 \times 2$ pixels $)$, which allowed them to estimate relative motion of adjacent arterial layers [4]. Recently, Zahnd et al. [5] have used intermediate sized blocks, namely $1.5 \times 0.3 \mathrm{~mm}(50 \times 10$ pixels $)$, and the sum of squared differences as the matching criterion, to estimate radial and longitudinal displacements of the proximal and distal arterial walls in healthy volunteers and diabetic subjects. The method of Bang et al. [6] produced a dense displacement vector map, from which 29 parameters were estimated representing amplitude, stretch/compression, and shear motion of the atherosclerotic plaque [7]. Motion of the carotid atheromatous plaque from 3-D ultrasound images has been estimated using OF [8]. Significant differences were found in relative plaque motion between symptomatic and asymptomatic subjects. Mokhtari-Dizaji et al. [9] used an OF-based motion analysis algorithm to estimate systolic and diastolic diameters of the right common carotid artery from sequences of B-mode and color Doppler ultrasound images. Murray et al. [10] combined OF with an amplitude modulation method and demonstrated its superiority over its combination with frequency modulation as well as over conventional $\mathrm{OF}$.

The use of different motion analysis techniques leads to different types of results, which may affect interpretation of findings 
and formulation of diagnosis. A preliminary study comparing $\mathrm{BM}$ with OF based on the Horn \& Schunck method $\left(\mathrm{OF}_{\mathrm{HS}}\right)$ showed that the shapes of the waveforms produced with the two techniques were different, although peaks occurred at the same time points [11]. Furthermore, additional algorithms, such as weighted least-squares $\mathrm{OF}\left(\mathrm{OF}_{\mathrm{LK}(\mathrm{WLS})}\right)$ or the affine block motion model (ABMM), an emerging method allowing not only translation but also rotation and scaling, may be investigated for suitability in estimating carotid artery wall motion using B-mode ultrasound.

While different techniques suitable for carotid artery wall motion estimation continue to appear, the literature lacks a quantitative evaluation of existing methods. In previous studies, the methods used were apparently selected at random among a large number of techniques in the image analysis literature. Therefore, in this paper, four well-known motion analysis techniques were investigated in terms of the estimation of motion of the carotid artery wall using 2-D B-mode ultrasound, in an attempt to suggest an optimal methodology for this application. $\mathrm{OF}_{\mathrm{HS}}$, $\mathrm{OF}_{\mathrm{LK}(\mathrm{WLS})}, \mathrm{BM}$, and ABMM were compared on the basis of experiments using simulated (computer-generated) data. Real ultrasound image sequences were then used to demonstrate the applicability of the selected motion analysis method.

\section{THEORETICAL CONCEPTS}

The estimation of the 2-D motion between two frames in a sequence is an ill-posed problem because its solution is not unique. The most commonly used equation in this problem is known as the gradient constraint equation and relates the velocity to the space-time derivatives at any one point of an image [12]

$$
\mathbf{u} \cdot \nabla I(\mathbf{x}, t)+I_{t}(\mathbf{x}, t)=0
$$

where $I(\mathbf{x}, t)$ is the image intensity as a function of space $\mathbf{x}=(x, y)$ and time $t, \mathbf{u}=(\Delta \mathbf{x} / \Delta t)=\left(u_{x}, u_{y}\right), \nabla I(\mathbf{x}, t)=\left(I_{x}, I_{y}\right)$, and $I_{t}$ denote spatial and temporal partial first derivatives, respectively, of the image $I$. Velocity cannot be readily recovered from (1) because it is an equation with two unknowns, $u_{x}$ and $u_{y}$.

$\mathrm{OF}$ the four motion estimation methods benchmarked in this study, $\mathrm{OF}_{\mathrm{HS}}$ and $\mathrm{BM}$ have been previously used to estimate carotid artery wall and plaque motion using ultrasound [3]-[9].

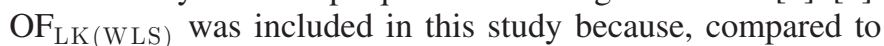
the more conventional $\mathrm{OF}_{\mathrm{HS}}$, it allows further smoothing out of large velocity differences between adjacent sites, which may be desirable in noisy images such as ultrasound images. Due to its local rigidity principles, $\mathrm{OF}_{\mathrm{LK}(\mathrm{WLS})}$ may be considered as a combination of differential $\mathrm{OF}$ and $\mathrm{BM}$ characteristics. Compared to conventional BM, the ABMM allows deformation of the interrogated block, which may be suitable for the nonrigid deformation experienced by the arterial wall.

\section{OF Based on the Horn \& Schunck Method}

$\mathrm{OF}$ is the term used to describe the velocity field generated by the relative motion between an object and the camera in a sequence of frames [13]. To estimate $\mathrm{OF}_{\mathrm{HS}}$ from (1), an additional constraint regarding the smoothness of the velocity field needs to be determined. The smoothness constraint requires that the energy of the image derivatives is minimal. Combining the two constraints, i.e., gradient constraint and velocity smoothness, results in an optimization problem where the optimal velocity minimizes the functional

$$
\begin{aligned}
J\left(u_{x}, u_{y}\right)= & \iint\left(I_{x} u_{x}+I_{y} u_{y}+I_{t}\right)^{2} d x d y \\
& +\lambda\left(\iint\left(u_{x x}^{2}+u_{x y}^{2}\right) d x d y\right. \\
& \left.+\iint\left(u_{y x}^{2}+u_{y y}^{2}\right) d x d y\right)
\end{aligned}
$$

where $\lambda$ is the regularization parameter which determines the effect of the smoothness constraint and $u_{i j}=\partial u_{i} / \partial j, i, j=x, y$ are the spatial derivatives of $u_{x}$ and $u_{y}$.

\section{Weighted Least-Squares OF}

A common way to constrain velocity is to use gradient constraints from neighboring pixels, assuming they share the same 2-D velocity. In reality, there may be no single velocity value that simultaneously satisfies all regional pixels, so the velocity that minimizes the constraint errors is found instead [14]. The least-squares estimator that minimizes the squared errors is

$$
E(\mathbf{u})=\sum_{\mathbf{x}} g(\mathbf{x}) \cdot\left[\mathbf{u} \cdot \nabla I(\mathbf{x}, t)+I_{t}(\mathbf{x}, t)\right]^{2}
$$

where $g(\mathbf{x})$ is a 2-D Gaussian weighted function, which is used to enhance constraints in the center of the neighborhood, thus increasing their influence. This is a modification with respect to the conventional Lucas-Kanade method [14], which does not use a weighted function in the least-squares estimator; more detail about $\mathrm{OF}_{\mathrm{LK}(\mathrm{WLS})}$ can be found in [12].

\section{$B M$}

Given a block of pixels, or reference block, in the first of two images, matching consists in finding the block in the second image that best matches the block in the first image, termed reference image [15]. This definition relies on the assumption that the reference block remains constant over time and motion which is valid if the frame rate is sufficiently high. The method requires a good measure of match, with large values when the selected block and the interrogated image region coincide in intensity levels and small otherwise. The normalized correlation coefficient corrected for the average values [3] was selected here as matching criterion. The search for the best-matched block is typically constrained to a search window, the size of which has to be appropriately chosen. A large search window allows accurate tracking of rapid movements that could be lost with a smaller window, but increases the possibility for mismatch and the algorithm's computational complexity.

\section{ABMM}

In the $\mathrm{BM}$ and $\mathrm{OF}_{\mathrm{LK}(\mathrm{WLS})}$ methods, the 2-D velocities are considered constant within pixel blocks. To overcome this constraint, the ABMM allows the block to undergo not only translation but also rotation and scaling [12]. An affine velocity field centered at location $\mathbf{x}_{0}=\left(x_{0}, y_{0}\right)$ can be expressed in matrix form as

$$
\mathbf{u}=\mathbf{A} \cdot \mathbf{c}
$$


where $\mathbf{c}=\left(c_{1}, c_{2}, c_{3}, c_{4}, c_{5}, c_{6}\right)^{T}$ are the model parameters expressing translation $\left(c_{1}, c_{4}\right)$, and rotation and scaling $\left(c_{2}, c_{3}, c_{5}, c_{6}\right)$. The matrix $\mathbf{A}$ has the form

$$
\mathbf{A}=\left[\begin{array}{cccccc}
1 & 0 & x-x_{0} & y-y_{0} & 0 & 0 \\
0 & 1 & 0 & 0 & x-x_{0} & y-y_{0}
\end{array}\right] .
$$

Combining (1) and (4) yields the gradient constraint equation

$$
\nabla I(\mathbf{x}, t) \cdot \mathbf{A} \cdot \mathbf{c}+I_{t}(\mathbf{x}, t)=0 .
$$

The model parameter vector can be estimated from (6), according to the principles outlined in [12].

\section{METHODOLOGY}

\section{A. Simulated Temporal Image Sequences}

Two types of simulated image sequences were generated for the evaluation of the motion analysis methods, one using a real image as a template and various noise levels and the other using Field II, an ultrasound simulation package, which provides a useful framework to simulate ultrasound fields by incorporating realistic transducer features [16]. The first type of simulation allows the study of the behavior of the methods under controlled noise, whereas the second type of simulation allows testing the methods in a realistic ultrasonic field environment. Motion of both types of simulated image sequences was achieved through a mathematical model for arterial wall motion. In the following, the two simulations as well as the mathematical motion model are described.

1) Simulations Using a Real Image Template and Different Noise Levels: A sequence of 87 gray scale images $\left(\mathrm{S}_{0}\right)$, corresponding to three cardiac cycles, was generated using a real B-mode ultrasound image of a healthy carotid artery as a template. The simulated images were then corrupted with Gaussian noise, resulting in two additional image sequences with increasing noise levels: a sequence with signal-to-noise ratio (SNR) equal to $25 \mathrm{~dB}\left(\mathrm{~S}_{\mathrm{G} 25}\right)$ and a sequence with $\mathrm{SNR}$ equal to 15 $\mathrm{dB}\left(\mathrm{S}_{\mathrm{G} 15}\right)$. Two sequences were additionally created by corrupting $\mathrm{S}_{0}$ with speckle noise, one with SNR equal to $25 \mathrm{~dB}$ $\left(\mathrm{S}_{\mathrm{SP} 25}\right)$ and one with $\mathrm{SNR}$ equal to $15 \mathrm{~dB}\left(\mathrm{~S}_{\mathrm{SP} 15}\right)$. Fig. 1(a), (c) shows examples of images of the $\mathrm{S}_{0}, \mathrm{~S}_{\mathrm{SP} 15}$, and $\mathrm{S}_{\mathrm{G} 15}$ sequences, respectively.

2) Simulations Using a Real Image Template and Field II: Using Field II, 87 B-mode ultrasound images were generated from a sequence of scattering strength maps [16] according to the procedures described in [17]. Briefly, the first frame of the scattering map sequence was designed using a real end-diastole B-mode ultrasound image as a template [see Fig. 1(a)]. To create the anatomic phantom of the carotid artery, 100000 randomly distributed scatterers were used. To create a sequence of anatomic phantoms, the scattering map was deformed according to the mathematical model of arterial wall motion discussed in the next section. To generate the simulated sequence $\left(\mathrm{S}_{\mathrm{F}}\right)$ from the sequence of phantoms, a typical vascular transducer was specified: 5-MHz central frequency, 64-element linear array with Hanning apodization for both transmission and reception, and with single focusing in transmission and multiple focusing in reception. The sampling frequency of the scanning was set equal to $100 \mathrm{MHz}$. A total of 128 scanning lines were used and the resultant image size was $300 \times 250$ pixels. An exam-

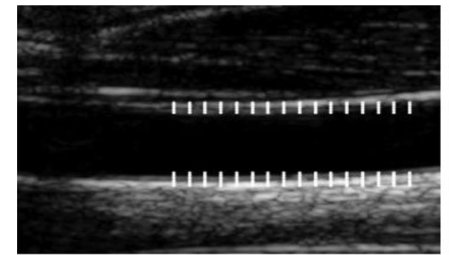

(a)

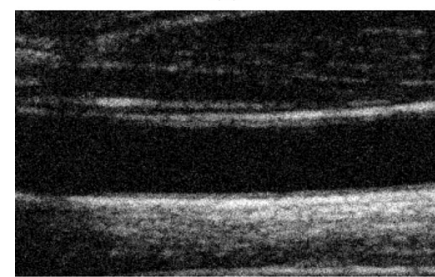

(c)

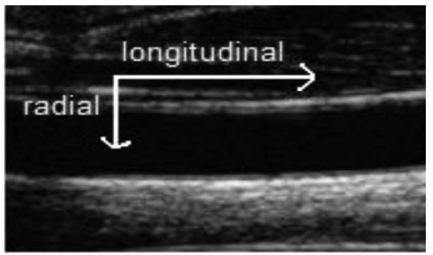

(b)

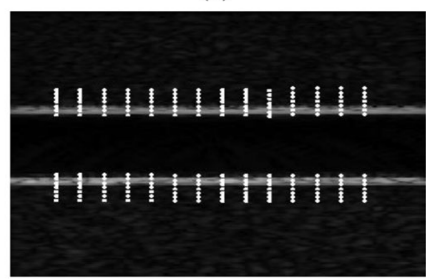

(d)
Fig. 1. Examples of images of the common carotid artery wall of synthetic sequences: (a) $\mathrm{S}_{0}$, (b) $\mathrm{S}_{\mathrm{SP} 15}$, (c) $\mathrm{S}_{\mathrm{G} 15}$, and (d) $\mathrm{S}_{\mathrm{F}}$. The white marks represent the selected pixels. $\mathrm{S}_{0}, \mathrm{~S}_{\mathrm{SP} 15}, \mathrm{~S}_{\mathrm{G} 15}$, and $\mathrm{S}_{\mathrm{F}}$ are defined in the text.

ple of the images of the simulated sequence $\left(\mathrm{S}_{\mathrm{F}}\right)$ is shown in Fig. 1(d).

3) Mathematical Model for Arterial Wall Motion: The mathematical model describing mechanical deformation of the arterial wall was defined as a separable model in space and time of the form

$$
\operatorname{def}(\mathbf{x}, t)=\left[\begin{array}{l}
r(\mathbf{x}, t) \\
l(\mathbf{x}, t)
\end{array}\right]=\left[\begin{array}{c}
r s(\mathbf{x}) \cdot r t(t) \\
l s(\mathbf{x}) \cdot l t(t)
\end{array}\right] .
$$

The definition of the terms $r(\mathbf{x}, t)$ and $l(\mathbf{x}, t)$ describing radial and longitudinal displacements, respectively, was based on a similar theoretical model defined for the intact left ventricle contraction [18]. The adoption of this model was based on the assumption that the deformation of individual parts of the cardiovascular system follows the same temporal rule.

Using this model, the temporal deformation $r t(t)$ of the carotid artery wall in the radial direction was defined in a piecewise fashion using a pulse function $\Pi\left(t_{i}, t_{i+1}\right)$

$$
r t(t)=\Pi\left(t_{0}, t_{1}\right) \cdot \sin ^{2} \frac{\pi \cdot t}{s \cdot t}+\Pi\left(t_{1}, t_{2}\right) \cdot(e+p \cdot t)
$$

where $s=3.5, T=29, e=1.146$, and $p=-0.4$.

The pulse functions $\Pi\left(t_{0}, t_{1}\right)$ and $\Pi\left(t_{1}, t_{2}\right)$ were defined as

$\Pi\left(t_{0}, t_{1}\right)=f_{1} \cdot \tanh \left(d\left(t-\left(t_{1}+k\right)\right)\right) \cdot\left(1+\tanh \left(d\left(t_{0}-t\right)\right)\right)$

$$
\Pi\left(t_{1}, t_{2}\right)=f_{2} \cdot\left(1+\tanh \left(d\left(t-t_{2}\right)\right)\right) \cdot\left(1+\tanh \left(d\left(t_{1}-t\right)\right)\right)
$$

where $f_{1}=f_{2}=0.25, d=1.22, t_{1}=0.25 \mathrm{~T}, k=0$, and $t_{2}=$ $0.95 \mathrm{~T}$.

Similar definitions were used for $l t(t)$ which was assumed to produce bidirectional longitudinal motion patterns, according to previously reported observations [4]. The parameter values used in (8)-(10) for $l t(t)$ were $s=0.65, T=29, e=7, p=$ $-0.2, f_{1}=1.9, d=2.07, t_{1}=0.39 \mathrm{~T}, k=0.10 \mathrm{~T}, f_{2}=0.25$, and $t_{2}=1.15 \mathrm{~T}$. All parameter values were determined based on experiments with real data [19].

The terms $r s(\mathbf{x}, t)$ and $l s(\mathbf{x}, t)$ express the spatial dependence of displacements and, because of motion weakening away from the lumen [3], they were defined as exponential functions of the 
radial position $y$

$$
\begin{aligned}
& r s(\mathbf{x})=a_{r} \cdot e^{b_{r}\left|y-y_{i}\right|} \\
& l s(\mathbf{x})=a_{l} \cdot e^{b_{l}\left|y-y_{i}\right|}
\end{aligned}
$$

where $y_{i}$ is the radial position of the lumen surface in the interrogated wall (anterior or posterior), $a_{r}=0.4033 \mathrm{~mm}$ and $\alpha_{l}=0.4069 \mathrm{~mm}$ correspond to the maximum amplitudes of radial and longitudinal displacements of the wall, respectively, and $b_{r}=-0.1722$ and $b_{l}=-1.075$ express the attenuation of the motion amplitude. The definition of (11) was based on the estimation of radial motion amplitude for a number of points in the carotid artery wall of a healthy subject. Note that $r s(\mathbf{x})$ is independent of the longitudinal position $x$. The parameters of (12) were determined using the values of shear strain (SS) in [4].

\section{B. Evaluation of Motion Analysis Methods}

1) Parameter Definition in Motion Analysis Methods: To apply the motion analysis methods described in Section II to ultrasound image sequences of the carotid artery, a number of parameters need to be determined. In $\mathrm{OF}_{\mathrm{HS}}$, the regularization parameter $\lambda$ was set to 1 . Experiments using the values $1,5,10$, 20 for $\lambda$ showed that the selected value resulted in the lowest error for different image areas and different noise levels. Based on similar experiments, a block size of $1.0 \times 1.5 \mathrm{~mm}(17 \times$ 25 pixels) [height $\times$ width] was used for $\mathrm{OF}_{\mathrm{LK}(\mathrm{WLS})}, \mathrm{BM}$, and ABMM; other block sizes interrogated were $0.5 \times 0.8(9 \times 13)$, $0.5 \times 1.5(9 \times 25)$, and $1.0 \times 0.8(17 \times 13) \mathrm{mm}$ (pixels). A larger value was selected for the width of the block, reflecting the fact that less relative motion is expected in the longitudinal direction. $\sigma_{x}$ and $\sigma_{y}$ of the Gaussian function used in $\mathrm{OF}_{\mathrm{LK}(\mathrm{WLS})}$ and $\mathrm{ABMM}$ were set equal to the width and height, respectively, of the reference block. The size of the search window in BM was set to $1.3 \times 1.3 \mathrm{~mm}(21 \times 21$ pixels $)$, based on maximal expected displacements as explained in [3]. Additionally, BM was implemented using the first image of a sequence as the reference image and within a double-step interpolation scheme [5], which leads to finer resolution in motion estimation without entailing high computational complexity.

2) Preprocessing and Selection of Image Areas: To compute continuous spatial $\left(I_{x}, I_{y}\right)$ and temporal $\left(I_{t}\right)$ gradients in $\mathrm{OF}_{\mathrm{HS}}$, $\mathrm{OF}_{\mathrm{LK}(\mathrm{WLS})}$, and the ABMM methods, the images were first smoothed using a Gaussian $7 \times 7$ kernel with standard deviation 0.8. Use of the Gaussian low-pass filter also allows the removal of high frequency noise inherent in ultrasound images which may be magnified by the use of derivatives. A Gaussian was preferred to alternative kernels because of its ability to approximate the convolution of different smoothing filters.

For the purposes of motion analysis using the four methods, image pixels at the wall-lumen interface were initially selected. Pixels at a distance of $1.5 \mathrm{~mm}$ along the interface (in the longitudinal direction) and at a distance of $0.45 \mathrm{~mm}$ through the tissue (in the radial direction) were then selected. Pixel density is lower in the longitudinal direction because less relative motion is expected compared to the radial direction. Reduction of the initially segmented area into individual pixels for further investigation reduces significantly the computational cost without compromising the related physiological information.
Fig. 1(a), (d) shows examples of pixels selection for the simulated sequences.

3) Performance of Motion Analysis Methods: The accuracy of motion estimation was evaluated using the warping index [18], defined as the mean geometric error in millimeters between the true and recovered displacements

$$
\varpi=\sqrt{\frac{1}{N_{R} M} \sum_{n \in M} \sum_{\mathbf{x} \in R}\left\|\mathbf{w}(n, \mathbf{x})-\mathbf{w}_{0}(n, \mathbf{x})\right\|^{2}}
$$

where $R$ is the region of interest (ROI), $N_{R}$ is the number of pixels in this region, $M$ is the total number of images in the sequence, and $\mathbf{w}$ and $\mathbf{w}_{0}$ correspond to the estimated and true displacements, respectively. This index represents an overall measure of the local error.

\section{Motion Analysis of Real Ultrasound Image Sequences of the Carotid Artery}

Temporal sequences of digitized B-mode ultrasound images of the carotid arteries of five young (25-32 years) and four elderly (44-73 years) healthy subjects as well as of 11 symptomatic (50-85 years, 50-90\% stenoses) and nine asymptomatic (52-90 years, 50-85\% stenoses) patients with atherosclerotic plaques were interrogated. For each subject, a scan sequence was recorded with an Advanced Technology Laboratory Ultramark 4 Duplex scanner and a high-resolution $7.5-\mathrm{MHz}$ linear array scan head. Carotid arteries were imaged in longitudinal sections using the following scanner settings: dynamic range, $60 \mathrm{~dB}$; 2-D gray map, linear; persistence, low; and frame rate, high. The sequences were recorded at a rate of 25 frames/s for $3 \mathrm{~s}$ (two to three cardiac cycles) during breath holding.

To study the radial and longitudinal movements in these image sequences, four ROIs were selected for each normal subject and five ROIs for each plaque patient. Specifically, two ROIs, namely AW and PW, were selected at opposite wall-lumen interfaces and one ROI, namely MA, was selected at the mediaadventitia interface of the posterior wall. Additionally, one ROI, namely PW2, was positioned distal to PW in the healthy subjects, and two ROIs, namely $\mathrm{Pt}$ and $\mathrm{Pb}$, were positioned at the top surface and the bottom of the plaque, respectively, in the patients. Examples of real images and selected ROIs are pre-

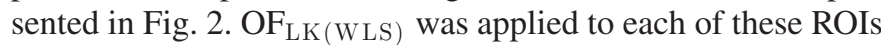
to produce radial and longitudinal displacement waveforms.

The following equations were used to estimate radial strain (RS), longitudinal strain (LS), and SS from the radial and longitudinal displacement waveforms:

$$
\begin{array}{r}
\mathrm{RS}=\frac{\left(\mathrm{RP}_{1}-\mathrm{RP}_{1(\mathrm{ed})}\right)-\left(\mathrm{RP}_{2}-\mathrm{RP}_{2(\mathrm{ed})}\right)}{\mathrm{RP}_{1(\mathrm{ed})}-\mathrm{RP}_{2(\mathrm{ed})}} \\
\mathrm{LS}=\frac{\left(\mathrm{LP}_{1}-\mathrm{LP}_{1(\mathrm{ed})}\right)-\left(\mathrm{LP}_{2}-\mathrm{LP}_{2(\mathrm{ed})}\right)}{\mathrm{LP}_{1(\mathrm{ed})}-\mathrm{LP}_{2(\mathrm{ed})}} \\
\mathrm{SS}=\arctan \left\{\frac{\left(\mathrm{LP}_{1}-\mathrm{LP}_{1(\mathrm{ed})}\right)-\left(\mathrm{LP}_{2}-\mathrm{LP}_{2(\mathrm{ed})}\right)}{\mathrm{RP}_{1(\mathrm{ed})}-\mathrm{RP}_{2(\mathrm{ed})}}\right\}
\end{array}
$$

where RP and LP denote radial and longitudinal displacements, respectively, for a pair of ROIs, and (ed) corresponds to 


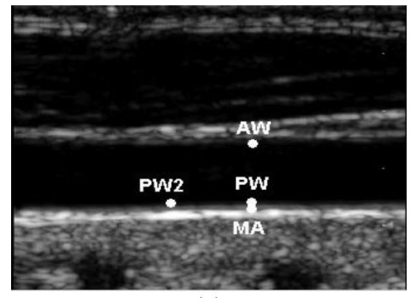

(a)

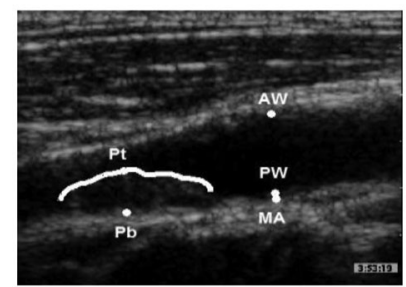

(c)

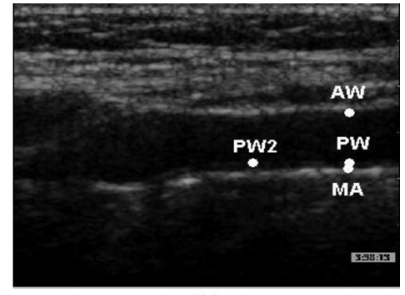

(b)

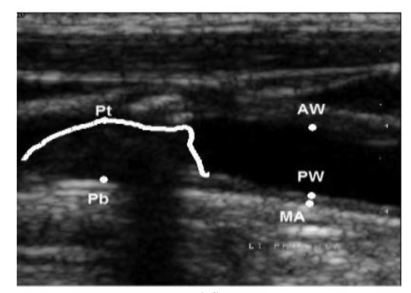

(d)
Fig. 2. First frames of ultrasound image sequences of (a) a young normal subject, (b) an elderly normal subject, (c) a symptomatic patient, and (d) an asymptomatic patient. The white marks indicate the centers of the selected ROIs. In (c) and (d), the plaque outlines for which MSV and MDSV were estimated are also shown.

end-diastole. Based on the aforementioned equations, the following strain waveforms were calculated.

1) RS at wall $\left(\mathrm{RS}_{W}\right)$, using (14) for $\mathrm{AW}$ and PW.

2) LS, using (15) for PW and PW2 for the healthy subjects and $\mathrm{PW}$ and $\mathrm{Pt}$ for the patients.

3) SS at wall ( $\left.\mathrm{SS}_{W}\right)$, using (16) for PW and MA.

4) $\mathrm{SS}$ at plaque $\left(\mathrm{SS}_{P}\right)$, using (16) for $\mathrm{Pt}$ and $\mathrm{Pb}$.

5) $\mathrm{RS}$ at plaque $\left(\mathrm{RS}_{P}\right)$, using (14) for $\mathrm{Pt}$ and $\mathrm{Pb}$.

Strain indices were then calculated from the previously described strain waveforms. Specifically, for each subject, the maximal absolute values of the strain waveforms were measured for each cardiac cycle, and $\mathrm{RSI}_{W}$ (radial strain index at wall), LSI (longitudinal strain index), $\mathrm{SSI}_{W}$ (shear strain index at wall), $\operatorname{SSI}_{P}$ (shear strain index at plaque), and $\mathrm{RSI}_{P}$ (radial strain index at plaque) were defined as the mean values of the corresponding measurements for all recorded cycles.

Two additional indices were estimated in the plaque cases, namely the maximal surface velocity (MSV) and the maximal discrepant surface velocity (MDSV), which were introduced in [8]. To this end, the surface of the plaque was manually defined. Examples of plaque surfaces are shown in Fig. 2 for a symptomatic and an asymptomatic case.

\section{RESULTS}

Table I shows the values of $\varpi$, in pixels, for the four methods for the sequences $\mathrm{S}_{0}, \mathrm{~S}_{\mathrm{G} 25}, \mathrm{~S}_{\mathrm{SP} 25}, \mathrm{~S}_{\mathrm{G} 15}, \mathrm{~S}_{\mathrm{SP} 15}$, and $\mathrm{S}_{\mathrm{F}}$, separately for the radial (RD), longitudinal (LD) and total (TD) displacements. To convert the reported values in millimeters, one can use the image resolution, which is 10 pixels $/ \mathrm{mm}$ for $\mathrm{S}_{\mathrm{F}}$ and 15.8 pixels $/ \mathrm{mm}$ for the other sequences, for both directions of motion.

According to the performance evaluation findings presented in Table I, the lowest warping indices were generally found for $\mathrm{OF}_{\mathrm{LK}(\mathrm{WLS})}$ followed by those for ABMM. The statistical significance of that difference was validated by estimating the root-mean-square (RMS) errors for total displacements for all
TABLE I

WARPING INDICES, IN PIXELS, FOR $\mathrm{OF}_{\mathrm{HS}}, \mathrm{OF}_{\mathrm{LK}(\mathrm{WLS})}$, BM, AND ABMM FOR THE SEQUENCES $\mathrm{S}_{0}, \mathrm{~S}_{\mathrm{G} 25}, \mathrm{~S}_{\mathrm{SP} 25}, \mathrm{~S}_{\mathrm{G} 15}, \mathrm{~S}_{\mathrm{SP} 15}$, AND $\mathrm{S}_{\mathrm{F}} \cdot \mathrm{RD}$ : RADIAL DISPLACEMENT; LD: LONGITUDINAL DISPLACEMENT; TD: TOTAL DISPLACEMENT

\begin{tabular}{cccccccc}
\hline \hline & & $\mathbf{S}_{\mathbf{0}}$ & $\mathbf{S}_{\mathbf{G 2 5}}$ & $\mathbf{S}_{\mathbf{S P 2 5}}$ & $\mathbf{S}_{\mathbf{G 1 5}}$ & $\mathbf{S}_{\mathbf{S P 1 5}}$ & $\mathbf{S}_{\mathbf{F}}$ \\
\hline $\mathbf{O F}_{\mathbf{H S}}$ & $R D$ & 1.90 & 4.71 & 8.94 & 9.10 & 14.36 & 2.16 \\
& $L D$ & 5.59 & 6.19 & 9.75 & 16.16 & 9.43 & 6.41 \\
& $T D$ & 5.91 & 7.77 & 13.22 & 18.55 & 17.17 & 6.78 \\
\hline $\mathbf{O F}_{\mathbf{L K}(\mathbf{W L S})}$ & $R D$ & 0.58 & 0.71 & 0.41 & 1.41 & 0.55 & 1.14 \\
& $L D$ & 0.84 & 1.69 & 0.74 & 2.16 & 1.64 & 2.20 \\
& $T D$ & 1.03 & $\mathbf{1 . 8 3}$ & $\mathbf{0 . 8 5}$ & $\mathbf{2 . 5 7}$ & $\mathbf{1 . 7 4}$ & $\mathbf{2 . 4 8}$ \\
\hline $\mathbf{B M}$ & $R D$ & 0.39 & 0.41 & 0.39 & 4.68 & 3.06 & 0.13 \\
& $L D$ & 0.81 & 7.58 & 1.71 & 17.44 & 7.33 & 3.24 \\
& $T D$ & $\mathbf{0 . 8 8}$ & 7.58 & 2.86 & 18.07 & 7.93 & 3.24 \\
\hline $\mathbf{A B M M}$ & $R D$ & 0.58 & 0.73 & 0.52 & 1.55 & 0.65 & 0.95 \\
& $L D$ & 0.90 & 1.90 & 0.88 & 2.35 & 1.80 & 3.12 \\
& $T D$ & 1.07 & 2.02 & 1.03 & 2.81 & 1.91 & 3.22 \\
\hline \hline Bon
\end{tabular}

Boldface indicates minimal warping indices for TD for each sequence.

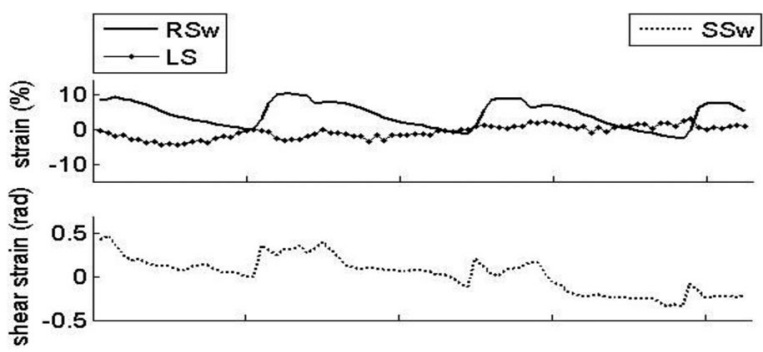

(a)

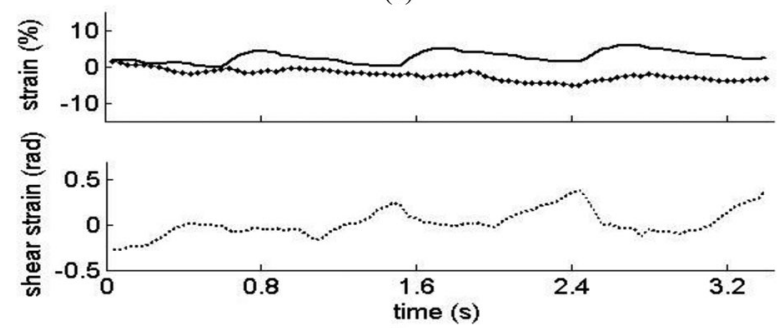

(b)

Fig. 3. Strain waveforms for (a) a young and (b) an elderly normal subject. $\mathrm{RS}_{W}$ : radial strain at wall; LS: longitudinal strain; $\mathrm{SS}_{W}$ : shear strain at wall.

interrogated pixels, resulting in two RMS error distributions for each sequence. Subsequently, a Wilcoxon rank-sum test between the error distributions of $\mathrm{ABMM}$ and $\mathrm{OF}_{\mathrm{LK}(\mathrm{WLS})}$ indicated the statistically significant superiority of the latter in most cases ( $p$-values: $0.39,0.031,0.035,0.030,0.039$, and 0.011 for $\mathrm{S}_{0}, \mathrm{~S}_{\mathrm{G} 25}, \mathrm{~S}_{\mathrm{SP} 25}, \mathrm{~S}_{\mathrm{G} 15}, \mathrm{~S}_{\mathrm{SP} 15}$, and $\mathrm{S}_{\mathrm{F}}$, respectively) The computational cost was marginally lower for $\mathrm{OF}_{\mathrm{LK} \text { (WLS) }}$ compared to ABMM; tracking one target pixel in an 87-image sequence required $28 \mathrm{~s}$ for $\mathrm{OF}_{\mathrm{LK}(\mathrm{WLS})}$ and $30 \mathrm{~s}$ for $\mathrm{ABMM}$, using a Pentium(R) dual-core CPU T4400 at $2.20 \mathrm{GHz}$. The corresponding costs for $\mathrm{OF}_{\mathrm{HS}}$ and $\mathrm{BM}$ were $0.9 \mathrm{~s}$ and $18 \mathrm{~s}$, respectively.

Fig. 3 shows examples of strain waveforms $\left(\mathrm{RS}_{W}, \mathrm{LS}\right.$, and $\mathrm{SS}_{W}$ ) for a young and an elderly normal subject. Fig. 4 shows examples of strain waveforms $\left(\mathrm{RS}_{W}, \mathrm{LS}, \mathrm{SS}_{W}, \mathrm{SS}_{P}\right.$, and $\mathrm{RS}_{P}$ ) for a symptomatic and an asymptomatic atherosclerotic patient. It is pointed out that LS corresponds to longitudinal strain between two ROIs in the wall in the normal subjects, but between a ROI on the wall and a ROI on the plaque in the plaque subjects. 


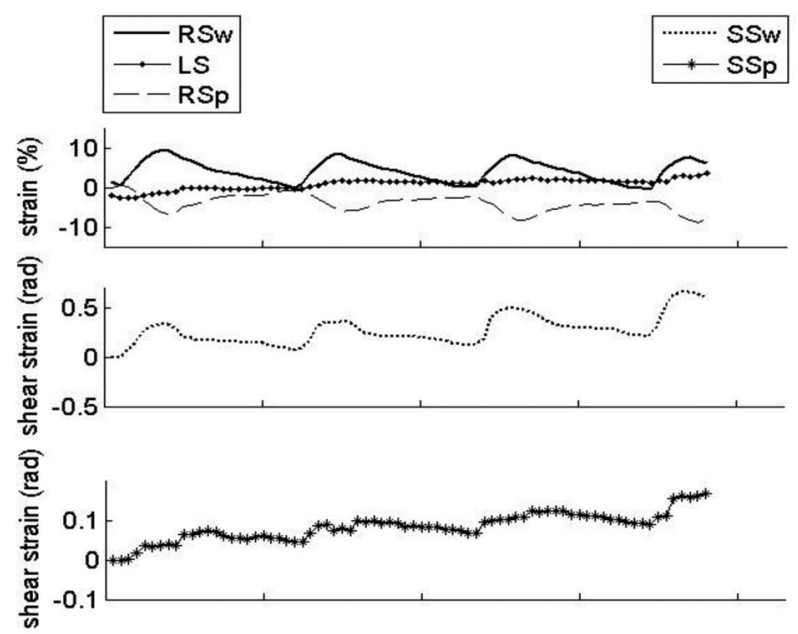

(a)

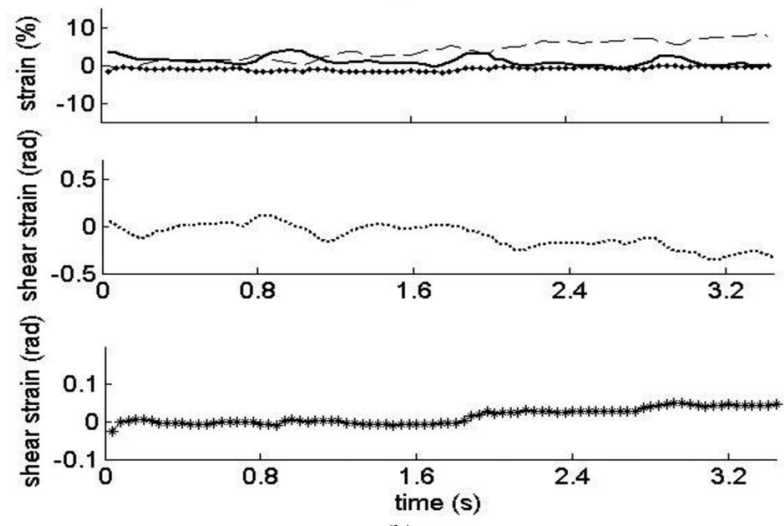

(b)

Fig. 4. Strain waveforms for (a) a symptomatic and (b) an asymptomatic atherosclerotic patient. $\mathrm{RS}_{W}$ : radial strain at wall; $\mathrm{LS}$ : longitudinal strain; $\mathrm{RS}_{P}$ : radial strain at plaque; $\mathrm{SS}_{W}$ : shear strain at wall; $\mathrm{SS}_{P}$ : shear strain at plaque.

As we can see, $\mathrm{RS}_{W}$ consistently exhibits a distinct periodic pattern, which seems to be of reduced amplitude in elderly subjects; this agrees with existing knowledge in arterial physiology [1]. $\mathrm{SS}_{W}$ also presents a periodic pattern of similar periodicity to that of RS. However, RS and SS are not always synchronized. LS in these examples shows lower amplitudes than RS and its periodicity is not always obvious. An interesting finding is that the RS of the symptomatic plaque [see Fig. 4(a), top graph] presents maximal values at minimal values of $\mathrm{RS}_{W}$. This suggests that the plaque is compressed at systole and expanded at diastole, which is an indication of a soft, compliant plaque.

Table II shows mean $( \pm$ sd) values of strain indices for the healthy subjects and the patients, respectively. As we can see, $\mathrm{RSI}_{W}$ was significantly lower in elderly subjects compared to younger ones. No statistical significance was found for the other parameters neither between normal young and normal elderly subjects nor between symptomatic and asymptomatic patients. In the same table, values for MSV and MDSV are also presented. Additional material including the outlines and displacement fields for all plaques investigated, as well as their demographic, clinical, and kinematic data, can be found at the authors' website http://biosim.ntua.gr/TITB-00435-2011_supplement.pdf.
TABLE II

MEAN ( \pm SD) VALUES OF STRAIN PARAMETERS OF THE CAROTID ARTERY OF

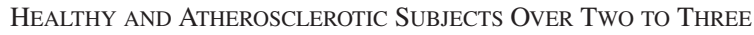
CONSECUTIVE CARDIAC CYCLES

\begin{tabular}{|c|c|c|}
\hline Strain indices & & \\
\hline \multicolumn{3}{|c|}{ A. Normal subjects } \\
\hline & Young $(n=5)$ & Elderly $(n=4)$ \\
\hline $\mathrm{RSI}_{W}(\%)$ & $12.23 \pm 2.44$ & $3.74 \pm 1.78^{*}$ \\
\hline LSI $(\%)$ & $3.96 \pm 1.68$ & $4.40 \pm 2.43$ \\
\hline $\mathrm{SSI}_{W}(\mathrm{rad})$ & $0.52 \pm 0.21$ & $0.37 \pm 0.15$ \\
\hline \multicolumn{3}{|c|}{ B. Atherosclerotic subjects } \\
\hline $\mathrm{RSI}_{W}(\%)$ & $5.09 \pm 1.73$ & $5.50 \pm 2.90$ \\
\hline LSI $(\%)$ & $2.79 \pm 1.71$ & $2.15 \pm 1.36$ \\
\hline $\mathrm{SSI}_{W}(\mathrm{rad})$ & $0.39 \pm 0.18$ & $0.37 \pm 0.20$ \\
\hline $\operatorname{SSI}_{P}(\mathrm{rad})$ & $0.07 \pm 0.04$ & $0.10 \pm 0.07$ \\
\hline $\mathrm{RSI}_{P}(\%)$ & $3.33 \pm 1.81$ & $4.32 \pm 2.34$ \\
\hline $\operatorname{MSV}(\mathrm{mm} / \mathrm{s})$ & $5.10 \pm 1.72$ & $6.22 \pm 2.19$ \\
\hline $\operatorname{MDSV}(\mathrm{mm} / \mathrm{s})$ & $4.92 \pm 1.66$ & $5.92 \pm 2.29$ \\
\hline
\end{tabular}

* Indicates statistically significant difference from young subjects (Wilcoxon rank-sum test, p-value $<0.05$ ).

\section{DISCUSSION AND CONCLUSION}

In this study, four techniques were evaluated in terms of motion analysis of the carotid artery wall from ultrasound images. In a set of experiments using customized synthetic images, $\mathrm{OF}_{\mathrm{LK}(\mathrm{WLS})}$ was found to outperform the other techniques and was subsequently applied to real data, including atherosclerotic and nonatherosclerotic cases. Waveforms as well as strain and kinematic indices were subsequently estimated to quantify the mechanical behavior of the arterial wall in the radial and longitudinal directions.

The study was undertaken in an attempt to address the observed lack of quantitative evaluation of existing methods in the literature. It is believed that the comparative evaluation undertaken will be proven valuable in the design of future studies in the continuously evolving field of ultrasound-image-based arterial wall motion analysis, because it will facilitate 1) selection of a specific method for motion analysis and 2) evaluation of alternative and/or emerging methods, within the proposed framework.

The methods investigated were tested on synthetic sequences produced using an ultrasound simulation package, namely Field II, which allows the evaluation not only of transducers and focusing schemes but also of image processing methods. Field II was used to evaluate the accuracy of a spatiotemporal nonrigid registration algorithm for ultrasound cardiac motion estimation [18]. Alternatively, validation of motion analysis algorithms can be performed using specially devised phantoms, which simulate the acoustic properties of biological tissue [6]. Although tissue-mimicking phantoms are useful for validation experiments, the required specialized equipment may not be easily available.

Algorithms were benchmarked on noise free as well as on noisy sequences. As expected, in the presence of increased noise $(15 \mathrm{~dB})$, all algorithms exhibited lowest performances. Noise reduces image contrast and produces fuzzy appearances in boundaries of anatomical structures, such as wall layers, thus compromising efficient region tracking. It is pointed out that noise is more pronounced in ultrasound images of elderly 
subjects as well as in plaque areas. This can also be observed in the examples of Fig. 2.

The warping indices produced with the different techniques

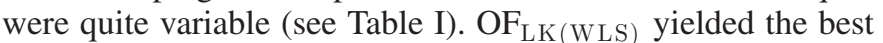
performance in most sequences and was closely followed by ABMM, a relatively new method. BM exhibited acceptable performance in the noiseless case $\left(\mathrm{S}_{0}\right)$, somewhat worse performance in the presence of medium noise $(25 \mathrm{~dB})$, and worst performance in the presence of severe noise $(15 \mathrm{~dB}) . \mathrm{OF}_{\mathrm{HS}}$ presented the lowest performance, which renders it prohibitive for use in this particular application. Based on these findings, $\mathrm{OF}_{\mathrm{LK}(\mathrm{WLS})}$, or, alternatively, ABMM would be the reasonable choices for motion analysis of the carotid artery wall from B-mode ultrasound.

Errors were higher in the longitudinal direction for all algorithms. This may be due to the fact that characteristic patterns are found in the radial direction because of different tissue areas and, therefore, different image intensities; such characteristic patterns facilitate region tracking in this direction. On the other hand, characteristic intensity patterns are not found in the longitudinal direction.

The incorporation of Kalman filtering in BM has been recently proposed in an attempt to enhance the performance of conventional BM [20]. In the same set of synthetic sequences as the one used in the present study, the warping indices produced with such combination were somewhat higher than those in this study. It is noted, however, that in [20], no interpolation was applied in the tracking algorithms.

The application of $\mathrm{OF}_{\mathrm{LK} \text { (WLS) }}$ in real ultrasound image data produced interesting findings potentially useful in studies of arterial physiology and pathophysiology. Waveforms of RS, LS, and SS (see Figs. 3 and 4) may reveal important aspects of the mechanical behavior of the normal and atherosclerotic arterial wall, such as anisotropy, displacement synchronization, etc.

Compared to previous studies reporting strain and kinematic indices of the arterial wall, our findings presented similar values in most cases. In the normal subject group, $\mathrm{SSI}_{W}$ values were not statistically different to those in [4], namely $0.59 \pm 0.21 \mathrm{rad}$ for young subjects (versus $0.42 \pm 0.26 \mathrm{rad}$ in [4]) and $0.37 \pm$ $0.15 \mathrm{rad}$ for elderly subjects (versus $0.30 \pm 0.17 \mathrm{rad}$ in [4]). In the plaque cases, MSV and MDSV were not statistically different to those reported in [8], in the symptomatic cases. In the asymptomatic subjects, however, MSV and MDSV were significantly lower in [8].

In conclusion, this study has demonstrated the ability of $\mathrm{OF}_{\mathrm{LK}(\mathrm{WLS})}$, a method that combines characteristics of BM and differential $\mathrm{OF}$, to outperform $\mathrm{OF}_{\mathrm{HS}}, \mathrm{BM}$, and $\mathrm{ABMM}$ in terms of synthetic data experiments, and to efficiently estimate RS, LS, and SS of healthy and diseased arterial wall areas. Systematic application of the method to larger datasets would provide useful insights into the mechanisms of arterial physiology and pathophysiology.

\section{REFERENCES}

[1] F. Hansen, P. Mangell, B. Sonesson, and T. Länne, "Diameter and compliance in the human common carotid artery-variations with age and sex," Ultrasound Med. Biol., vol. 21, no. 1, pp. 1-9, 1995.
[2] C. G. Caro and K. H. Parker, "The effect of haemodynamic factors on the arterial wall," in Atherosclerosis, A. G. Olsson, Ed. Edinburgh U.K.: Churchill Livingstone, 1987, pp. 100-110.

[3] S. Golemati, A. Sassano, M. J. Lever, A. A. Bharath, S. Dhanjil, and A. N. Nicolaides, "Motion analysis of carotid atherosclerotic plaque from B-mode ultrasound," Ultrasound Med. Biol., vol. 29, no. 3, pp. 387-399, Mar. 2003.

[4] M. Cinthio, A. R. Ahlgren, J. Bergkvist, T. Jansson, H. W. Persson, and $\mathrm{K}$. Lindström, "Longitudinal movements and resulting shear strain of the arterial wall," Amer. J. Physiol._Heart Circul. Physiol., vol. 291, no. 1, pp. H394-H402, Jul. 2006.

[5] G. Zahnd, L. Boussel, A. Marion, M. Durand, P. Moulin, A. Sérusclat, and D. Vray, "Measurement of two-dimensional movement parameters of the carotid artery wall for early detection of arteriosclerosis: A preliminary clinical study," Ultrasound Med. Biol., vol. 37, no. 9, pp. 1421-1429, Sep. 2011.

[6] J. Bang, T. Dahl, A. Bruinsma, J. H. Kaspersen, T. A. N. Hernes, and H. O. Myhre, "A new method for analysis of motion of carotid plaques from RF ultrasound images," Ultrasound Med. Biol., vol. 29, no. 7, pp. 967-976, Jul. 2003.

[7] T. Dahl, J. Bang, A. Ushakova, S. Lydersen, and H. O. Myhre, "Parameters describing motion in carotid artery plaques from ultrasound examination: A reproducibility study," Ultrasound Med. Biol., vol. 30, no. 9, pp. 1133 1143, Sep. 2004.

[8] S. Meairs and M. Hennerici, "Four-dimensional ultrasonographic characterization of plaque surface motion in patients with symptomatic and asymptomatic carotid artery stenosis," Stroke, vol. 30, no. 9, pp. 18071813, Sep. 1999.

[9] M. Mokhtari-Dizaji, M. Montazeri, and H. Saberi, "Differentiation of mild and severe stenosis with motion estimation in ultrasound images," Ultrasound Med. Biol., vol. 32, no. 10, pp. 1493-1498, Oct. 2006.

[10] V. Murray, S. E. Murillo, C. P. Loizou, C. S. Pattichis, E. Kyriacou, and A. Nicolaides, "An AM-FM model for motion estimation in atherosclerotic plaque videos," in Proc. 41 st Asilomar Conf. Signals Syst. Comput., Nov. 4-7, 2007, pp. 746-750.

[11] J. Stoitsis, S. Golemati, A. Dimopoulos, and K. Nikita, "Analysis and quantification of arterial wall motion from B-mode ultrasound imagescomparison of block-matching and optical flow," in Proc. IEEE Eng. Med. Biol. Soc. Conf., 2005, vol. 5, pp. 4469-4472.

[12] D. Fleet and Y. Weiss, "Optical flow estimation," in Handbook of Mathematical Models in Computer Vision, N. Paragios, Y. Chen, and O. Faugeras, Eds. New York: Springer-Verlag, 2006.

[13] B. K. P. Horn and B. G. Schunck, "Determining optical flow," Artif. Intell., vol. 17, pp. 185-203, 1981.

[14] B. D. Lucas and T. Kanade, "An iterative image registration technique with an application to stereoscopic vision," in Proc. Int. Conf. Artif. Intell., 1981, pp. 674-679.

[15] F. Dufaux and F. Moscheni, "Motion estimation techniques for digital TV: A review and a new contribution," Proc. IEEE, vol. 83, no. 6, pp. 858-876, Jun. 1995.

[16] J. Jensen, "Field: A program for simulating ultrasound systems," Med. Biol. Eng. Comput., vol. 34, Suppl. 1, pp. 351-353, 1996.

[17] J. Stoitsis, S. Golemati, V. Koropouli, and K. S. Nikita, "Simulating dynamic B-mode image data of the common carotid artery," in Proc. IEEE Int. Workshop Imag. Syst. Tech., 2008, pp. 144-148.

[18] M. J. Ledesma-Carbayo, J. Kybic, M. Desco, A. Santos, M. Sühling, P. Hunziker, and M. Unser, "Spatio-temporal nonrigid registration for ultrasound cardiac motion," IEEE Trans. Med. Imag., vol. 24, no. 9, pp. 1113-1126, Sep. 2005.

[19] J. Stoitsis, S. Golemati, E. Bastouni, and K. S. Nikita, "A mathematical model of the mechanical deformation of the carotid artery wall and its application to clinical data," in Proc. IEEE Eng. Med. Biol. Soc. Conf., 2007, pp. 2163-2166.

[20] A. Gastounioti, S. Golemati, J. Stoitsis, and K. S. Nikita, "Comparison of Kalman-filter-based approaches for block matching in arterial wall motion analysis from B-mode ultrasound," Meas. Sci. Technol., vol. 22, no. 11, pp. 1-9, Nov. 2011.

Authors' photographs and biographies not available at the time of publication. 